\title{
Medium Effects on Binary Collisions with the $\Delta$ Resonance
}

\author{
T.-S. H. Lee \\ Physics Division, Argonne National Laboratory, Argonne, IL. 60439-4843
}

\begin{abstract}
To facilitate the relativistic heavy-ion calculations based on transport equations, the binary collisions involving a $\Delta$ resonance in either the entrance channel or the exit channel are investigated within a Hamiltonian formulation of $\pi N N$ interactions. An averaging procedure is developed to define a quasi-particle $\Delta^{*}$ and to express the experimentally measured $N N \rightarrow \pi N N$ cross section in terms of an effective $N N \rightarrow N \Delta^{*}$ cross section. In contrast to previous works, the main feature of the present approach is that the mass and the momentum of the produced $\Delta^{*}$ 's are calculated dynamically from the bare $\Delta \leftrightarrow \pi N$ vertex interaction of the model Hamiltonian and are constrained by the unitarity condition. The procedure is then extended to define the effective cross sections for the experimentally inaccessible $N \Delta^{*} \rightarrow N N$ and $N \Delta^{*} \rightarrow N \Delta^{*}$ reactions. The predicted cross sections are significantly different from what are commonly assumed in relativistic heavy-ion calculations. The $\Delta$ potential in nuclear matter has been calculated by using a BrucknerHartree-Fock approximation. By including the mean-field effects on the $\Delta$ propagation, the effective cross sections of the $N N \rightarrow N \Delta^{*}, N \Delta^{*} \rightarrow N N$ and $N \Delta^{*} \rightarrow N \Delta^{*}$ reactions in nuclear matter are predicted. It is demonstrated that the density dependence is most dramatic in the energy region close to the pion production threshold.
\end{abstract}


Pacs Numbers: 25.75.+r

Typeset using REVTEX 


\section{INTRODUCTION}

Nuclear matter with a high population of nucleon resonances $\left(\Delta, N^{*}\right.$ 's $)$ is expected to exist in some astrophysical objects and can be created in relativistic heavy-ion collisions. With the very dedicated experimental efforts at GSI, AGS and CERN in the past decade, it appears that the properties of such a nuclear system can now be investigated. Experimental evidence for creating a $\Delta$-rich nuclear system during relativistic heavy-ion collisions have recently been reported [1,2]. This interesting development has raised two theoretical issues. First, it is necessary to examine the extent to which the theoretical models employed in identifying the $\Delta$-rich matter are valid. In the calculations based on transport equations [3] 9], some assumptions were made to define the cross sections of binary collisions involving a $\Delta$ in either the entrance channel or the exit channel. This needs to be clarified within a rigorous formulation of the scattering theory with resonance excitations. The medium effects on $\Delta$ propagation assumed in those calculations must also be examined from a more fundamental point of view. One possibility is to calculate the $\Delta$ mean field using the well-developed nuclear many-body methods [10,14]. The second theoretical issue is how to develop a microscopic approach to understand the $\Delta$-rich matter in terms of the elementary $N N, N \Delta$ and $\Delta \Delta$ collisions and their coupling with the pion production channels. These two theoretical issues are closely related and must be addressed within the same theoretical framework. The heart of the problem is to define precisely and to determine quantitatively all possible binary collisions in the $\Delta$-rich matter. In this paper, we will address this problem by employing a Hamiltonian model of $\pi N N$ interactions which was previously developed to describe $N N$ scattering up to $2 \mathrm{GeV}$ [11] 13] and the $\Delta$-nucleus dynamics in pion-nucleus reactions [14]. Our main objective is to provide the theoretical input to the relativistic heavy-ion calculations using transport equations [3] 9].

In section II, we briefly review the coupled $N N \oplus N \Delta$ scattering equations within the $\pi N N$ Hamiltonian model developed in Refs. 1113. We derive formulas in section III to relate the experimentally observed $N N \rightarrow \pi N N$ reaction to the resonating $N \Delta$ state. An 
averaging procedure is then developed to define a quasi-particle $\Delta^{*}$ and to evaluate the cross sections for binary collisions with a $\Delta^{*}$ in either the entrance channel or the exit channel. In section IV, we recall the approach of Ref. [14] to calculate the mean-field effects on $\Delta$ propagation which are then used to calculate the medium-corrected cross sections. In section $\mathrm{V}$, we present our predictions of the cross sections for $N N \rightarrow N \Delta^{*}, N \Delta^{*} \rightarrow N N$ and $N \Delta^{*} \rightarrow N \Delta^{*}$ transitions in free space and in nuclear medium. A summary is given in section VI.

\section{HAMILTONIAN MODEL OF $\pi$ NN INTERACTIONS}

An important advance in intermediate energy physics is the development of a microscopic approach to understand the nuclear reactions induced by pions, protons, and electrons in terms of the interactions between $\pi, N$, and $\Delta$ degrees of freedom. Such an approach must start with a $\pi N N$ model which can describe the following elementary processes

$$
\begin{aligned}
\pi N & \rightarrow \pi N, \quad E_{L} \leq 300 \mathrm{MeV} \\
N N & \rightarrow N N, \\
& \rightarrow \pi N N, \quad E_{L} \leq 1000 \mathrm{MeV} ; \\
\pi d & \rightarrow \pi d, \\
& \rightarrow N N, \\
& \rightarrow \pi N N \quad E_{L} \leq 300 \mathrm{MeV} .
\end{aligned}
$$

The developments of $\pi N N$ models have been reviewed in Ref. [16]. In this work we employ the $\pi N N$ model developed in Ref. [11]13. The main feature of the constructed $\pi N N$ Hamiltonian is that the $N N \leftrightarrow N \Delta$ interaction can be directly constrained by data on the $N N \rightarrow N \Delta \rightarrow \pi N N$ reaction, and the $N \Delta \rightarrow N \Delta$ interaction can also be related to the $\pi d$ reactions. This allows a more realistic determination of the phenomenological parts of

the interactions involving the $\Delta$. For example, it was found [11] 13$]$ that the ranges of the $\pi N N$ and $\pi N \Delta$ vertices in the $N N \leftrightarrow N \Delta$ transition potential must be less than about 750 
$\mathrm{MeV}$ for a monopole form if the the $N N \rightarrow \pi N N$ reaction cross sections can be reasonably described. This phenomenological procedure is not avoidable in any meson-exchange model since a fundamental theory of short-range $N N$ and $N \Delta$ interactions is still not available.

In this first application of the $\pi N N$ model to relativistic heavy-ion collisions, we will focus on the $\Delta$ resonance and neglect the weaker non-resonant $\pi N$ interactions. The $\pi N N$ model Hamiltonian within the formulation of Refs. [11] can then be written as

$$
H=H_{0}+H_{\text {int }},
$$

where $H_{0}$ is the sum of free energy operators for the $N, \Delta$ and $\pi$ degrees of freedom, and

$$
\begin{aligned}
H_{\text {int }} & =\sum_{i=1}^{2}\left[h_{\pi N, \Delta}(i)+h_{\Delta, \pi N}(i)\right] \\
& +\frac{1}{2} \sum_{i, j=1}^{2}\left[V_{N N, N N}(i, j)+V_{N N, N \Delta}(i, j)+V_{N \Delta, N N}(i, j)+V_{N \Delta, N \Delta}(i, j)\right]
\end{aligned}
$$

Note that the vertex interactions $h_{\pi N, \Delta}$ and $h_{\Delta, \pi N}$ describe the $\pi N \leftrightarrow \Delta$ transition. They can renormalize the bare $\Delta$ mass and generate a $N \Delta \rightarrow \Delta N$ interaction due to the exchange of an "on-mass-shell" pion. In the following presentation, this $N \Delta \rightarrow \Delta N$ is included in $V_{N \Delta, N \Delta}$ of Eq. (5).

The derivation of the $\pi N N$ scattering equations from the above model Hamiltonian can be found in section II of Ref. [12]. By some straightforward derivations using Eqs. (3.27), (3.31), and (3.24) of Ref. [12], we obtain the following equations defined in the coupled $N N \oplus N \Delta$ space

$$
\begin{aligned}
& T_{N N, N N}(E)=\hat{V}_{N N, N N}(E)+\hat{V}_{N N, N N}(E) G_{N N}(E) T_{N N, N N}(E), \\
& T_{N \Delta, N N}(E)=\Omega_{N \Delta}^{(-)+}(E) V_{N \Delta, N N}\left[1+G_{N N}(E) T_{N N, N N}(E)\right], \\
& T_{N N, N \Delta}(E)=\left[1+T_{N N, N N}(E) G_{N N}(E)\right] V_{N N, N \Delta} \Omega_{N \Delta}^{(+)}(E), \\
& T_{N \Delta, N \Delta}(E)=t_{N \Delta, N \Delta}(E)+T_{N \Delta, N N} V_{N N, N \Delta} \Omega_{N \Delta}^{(+)}(E) .
\end{aligned}
$$

In the above equations, we have defined the propagators

$$
\begin{aligned}
G_{N N}(E) & =\frac{P_{N N}}{E-H_{0}+i \epsilon}, \\
G_{N \Delta}(E) & =\frac{P_{N \Delta}}{E-H_{0}-\Sigma(E)},
\end{aligned}
$$


where the $\Delta$ self-energy is determined by the $h_{\pi N \leftrightarrow \Delta}$ vertex interaction in the presence of a spectator nucleon

$$
\Sigma_{\Delta}(E)=\sum_{i=1}^{2} h_{\Delta, \pi N}(i) \frac{P_{\pi N N}}{E-H_{0}+i \epsilon} h_{\pi N, \Delta}(i) .
$$

$P_{N N}, P_{N \Delta}$, and $P_{\pi N N}$ are respectively the projection operators for the $N N, N \Delta$, and $\pi N N$ states.

The main feature of the above scattering formulation is that the effects due to the $V_{N \Delta, N \Delta}$ interaction are isolated in the $N \Delta$ scattering t-matrix $t_{N \Delta, N \Delta}$ defined by

$$
t_{N \Delta, N \Delta}(E)=V_{N \Delta, N \Delta}+V_{N \Delta, N \Delta} G_{N \Delta}(E) t_{N \Delta, N \Delta}(E)
$$

and the $N \Delta$ scattering operators defined by

$$
\begin{aligned}
& \Omega_{N \Delta}^{(+)}(E)=1+G_{N \Delta}(E) t_{N \Delta, N \Delta}(E) \\
& \Omega_{N \Delta}^{(-)+}(E)=1+t_{N \Delta, N \Delta}(E) G_{N \Delta}(E) .
\end{aligned}
$$

The $N \Delta$ scattering also has a contribution to the effective $N N$ potential in Eq. (6). Explicitly, we have

$$
\hat{V}_{N N, N N}(E)=V_{N N, N N}+U_{N N, N N}^{(2)}(E)
$$

with

$$
U_{N N, N N}^{(2)}(E)=V_{N N, N \Delta} G_{N \Delta}(E)\left[1+t_{N \Delta, N \Delta}(E) G_{N \Delta}(E)\right] V_{N \Delta, N N}
$$

The task is then to find an appropriate parameterization of the model Hamiltonian Eq. (5) to best reproduce the data of the $\pi N N$ processes listed in Eqs. (1)-(3). This was first achieved by Betz and Lee [17] using the separable parameterization. In this work we consider the meson-exchange parameterization of Refs. 111 13. It was found that the $\pi N N$ data can be best reproduced by using: (1) the vertex interaction $h_{\pi N \leftrightarrow \Delta}$ determined from fitting the $\pi N$ scattering phase shift in $P_{33}$ channel, (2) the one-pion-exchange model 18 of the transition potentials $V_{N N \leftrightarrow N \Delta}$ and $V_{N \Delta, N \Delta}$ with a monopole form factor of a cutoff 
parameter $\Lambda=650 \mathrm{MeV} / \mathrm{c}(3)$, the $N N$ interaction $V_{N N, N N}$ of Eq. (16) is defined by using a subtraction of the Paris potential [19]

$$
V_{N N, N N}=V_{\text {Paris }}-U_{N N, N N}^{(2)}\left(E=E_{s}\right)
$$

with $E_{s}=10 \mathrm{MeV}$ laboratory energy. Note that the above definition of the $N N$ interaction amounts to removing phenomenologically the two-pion-exchange with an intermediate $N \Delta$ state from the Paris potential, in order to avoid the double counting of the $N \Delta$ effect. This approach was also developed independently by Sauer and collaborators [20]. The details of the determination of the model Hamiltonian as well as comparisons with the $\pi N N$ data can be found in Refs. [11 [13]. Here we illustrate the validity of the model by showing in Fig. 1 that the predictions of the model are also in good agreement with the most recent np polarization data [15].

Before we proceed further, it is important to clarify the meaning of the $\Delta$ degree of freedom of the considered $\pi N N$ model. Because of the presence of the vertex interaction

$h_{\Delta \leftrightarrow \pi N}$, the $\Delta$ is certainly not a physical particle which can be detected experimentally. To investigate the $\Delta$ dynamics, we need to consider the $N N \leftrightarrow \pi N N$ and $\pi N N \rightarrow \pi N N$ reactions. The amplitudes of these reactions can be calculated from the vertex interaction $h_{\Delta \leftrightarrow \pi N}$ and the baryon-baryon t-matrix defined in Eqs. (6)-(9) by the following equations

$$
\begin{aligned}
T_{N N, \pi N N}(E) & =T_{N N, N \Delta}(E) G_{N \Delta}\left[\sum_{i=1}^{2} h_{\Delta, \pi N}(i)\right] \\
T_{\pi N N, N N}(E) & =\left[\sum_{i=1}^{2} h_{\pi N, \Delta}(i)\right] G_{N \Delta}(E) T_{N \Delta, N N}(E) \\
T_{\pi N N, \pi N N}(E) & =\left[\sum_{i=1}^{2} h_{\pi N, \Delta}(i)\right] G_{N \Delta}(E) T_{N \Delta, N \Delta}(E)\left[\sum_{i=1}^{2} h_{\Delta, \pi N}(i)\right] .
\end{aligned}
$$

\section{EFFECTIVE BINARY CROSS SECTIONS WITH $\Delta^{*}$}

We will only consider the transport equations of relativistic heavy-ion collisions which are determined by the cross sections of the collisions between "on-mass-shell" physical particles. 
Within the model defined by the $\pi N N$ Hamiltonian Eqs. (4)-(5), the transport equations can then only be written in terms of $\pi$ and $N$ variables. It is however possible to express the dynamics associated with the pion in terms of a quasi-particle $\Delta^{*}$ which has a mass equal to the invariant mass of a $\pi N$ subsystem. The transport equations can then be re-written in terms of $N$ and $\Delta^{*}$. In such a approach, the mass of the $\Delta^{*}$ can range from $m_{N}+m_{\pi}$ to the highest energy allowed by the collision energy. To carry out calculations, it is necessary to define the $N N \leftrightarrow N \Delta^{*}$ and $N \Delta^{*} \rightarrow N \Delta^{*}$ cross sections. In the following we will try to define rigorously these cross sections in terms of the $N N \leftrightarrow \pi N N$ and $\pi N N \leftrightarrow \pi N N$ amplitudes defined in the previous section.

To proceed, let us first consider the $N N \rightarrow \pi N N$ and the three-body kinematics in the center of mass frame (Fig. 2):

$$
\begin{aligned}
\vec{k} & =\vec{k}_{1}, \\
& =-\left(\vec{k}_{2}+\vec{k}_{3}\right) .
\end{aligned}
$$

For a given total collision energy $E$ and a given momentum $\vec{k}$, the invariant mass $M$ and the internal relative momentum $\vec{q}$ of the $\pi N(23)$ subsystem, called $\Delta^{*}$, are defined by the following relations

$$
\begin{aligned}
& E=E_{N}(k)+\left[M^{2}+k^{2}\right]^{1 / 2}, \\
& M=E_{N}(q)+E_{\pi}(q),
\end{aligned}
$$

where $E_{\alpha}(q)=\left(m_{\alpha}^{2}+q^{2}\right)^{1 / 2}$ is the energy of a particle with mass $m_{\alpha}$. Eq. (23) also leads to a useful relation

$$
M^{2}=E^{2}-2 E E_{N}(k)+m_{N}^{2}
$$

Clearly, Eqs. (24) and (25) imply that the invariant mass of the $\Delta^{*}$ is restricted to the region $\left(m_{N}+m_{\pi}\right) \leq M \leq\left(E-m_{N}\right)$. By using Eqs. (23)-(24), the momenta $k$ and $q$ can be written as 


$$
\begin{aligned}
k & =\frac{1}{2 E}\left[\left(E^{2}-m_{N}^{2}-M^{2}\right)^{2}-4 M^{2} m_{N}^{2}\right]^{1 / 2}, \\
q & =\frac{1}{2 M}\left[\left(M^{2}-m_{N}^{2}-m_{\pi}^{2}\right)^{2}-4 m_{N}^{2} m_{\pi}^{2}\right]^{1 / 2} .
\end{aligned}
$$

In terms of the kinematical variables defined above and in Fig. 2, the matrix element of the $N N \rightarrow \pi N N$ transition operator, Eq. (20), can be written (suppressing spin-isospin indices) as

$$
<\vec{k}, \vec{q}\left|T_{\pi N N, N N}(E)\right| \vec{k}_{0}>=h_{\pi N, \Delta}(\vec{q}) \frac{1}{E-E_{N}(k)-E_{\Delta}(k)-\Sigma(E, \vec{k})} T_{N \Delta, N N}\left(\vec{k}, \vec{k}_{0}, E\right) .
$$

We choose the normalization $\left\langle\vec{k}^{\prime} \mid \vec{k}\right\rangle=\delta\left(\vec{k}^{\prime}-\vec{k}\right)$. The scattering t-matrix is related to the S-matrix by (in operator form)

$$
S(E)=1-2 \pi i \delta\left(E-H_{0}\right) T(E) .
$$

We next define the following partial-wave expansions

$$
h_{\pi N, \Delta}(\vec{q})=y_{l_{23} s_{23}}^{s_{\Delta} m_{s_{\Delta}}}(\hat{q}) h_{\pi N, \Delta}(q)<s_{\Delta} m_{s_{\Delta}} \mid
$$

and

$$
T_{N \Delta, N N}\left(\vec{k}, \vec{k}_{0}, E\right)=\sum_{J, M_{J}} \sum_{L S, L_{\Delta} S_{\Delta}} y_{L_{\Delta} S_{\Delta}}^{J M_{J}}(\hat{k}) T_{L_{\Delta} S_{\Delta}, L S}^{J}\left(k, k_{0}, E\right) y_{L S}^{J M_{J}^{+}}\left(\hat{k}_{0}\right) .
$$

In the above equations, we have defined the spin-angular vector

$$
\begin{aligned}
y_{l s}^{j m}(\hat{p}) & =\sum_{m_{l} m_{s}} \sum_{m_{s_{1}} m_{s_{2}}} Y_{l m_{l}}(\hat{p})\left|s_{1} m_{s_{1}}>\right| s_{2} m_{s_{2}}> \\
& \times<l s m_{l} m_{s}\left|j m><s_{1} s_{2} m_{s_{1}} m_{s_{2}}\right| s m_{s}>,
\end{aligned}
$$

where $Y_{l m_{l}}(\hat{q})$ is the usual spherical harmonic function, $\left(s_{i}, m_{s_{i}}\right)$ are the spin variables for the i-th particle.

By using the definitions Eqs. (28)-(29) and the variables defined by Eqs. (23)-(27), we can write the total cross section of the two-step process $N N \rightarrow N \Delta \rightarrow N N \pi$ (Fig. 2) as

$$
\begin{aligned}
\sigma^{t o t}(E)= & \frac{1}{\left(2 s_{1}^{\prime}+1\right)\left(2 s_{2}^{\prime}+1\right)} \sum_{\text {spins }} \frac{(2 \pi)^{4}}{k_{0}^{2}}\left[\frac{k_{0} E_{N}\left(k_{0}\right)}{2}\right] \int d \vec{k} \int d \vec{q} \\
& \delta\left(E-E_{N}(k)-\sqrt{\left(E_{N}(q)+E_{\pi}(q)\right)^{2}+k^{2}}\right)\left|\sum_{m_{s_{\Delta}}}<m_{s_{2}} m_{s_{3}}\right| h_{\pi N, \Delta}(\vec{q}) \mid m_{s_{\Delta}}> \\
\times & \frac{1}{E-E_{N}(k)-E_{\Delta}(k)-\Sigma(k, E)}<m_{s_{\Delta}} m_{s_{1}}\left|T_{N \Delta, N N}\left(\vec{k}, \vec{k}_{0}, E\right)\right| m_{s_{1}}^{\prime} m_{s_{2}}^{\prime}>\left.\right|^{2},
\end{aligned}
$$


where the initial momentum $k_{0}$ is defined by the total energy $E=E_{N}\left(k_{0}\right)+E_{N}\left(k_{0}\right)$. The $\Delta$ self-energy is found to be

$$
\Sigma(k, E)=\int \frac{\left|h_{\Delta, \pi N}\left(q^{\prime}\right)\right|^{2} q^{\prime 2} d q^{\prime}}{\left[\left(E-E_{N}(k)\right)^{2}-k^{2}\right]^{1 / 2}-E_{N}\left(q^{\prime}\right)-E_{\pi}\left(q^{\prime}\right)+i \delta} .
$$

Substituting the expansions Eqs. (30)-(31) into Eq. (33) and integrating over angles $\hat{q}$ and $\hat{k}$, we obtain

$$
\sigma_{N N \pi \leftarrow N N}^{t o t}(E)=\sum_{J, L S, L_{\Delta} S_{\Delta}} d \sigma_{L_{\Delta} S_{\Delta}, L S}^{J}(E)
$$

with

$$
d \sigma_{L_{\Delta} S_{\Delta}, L S}^{J}(E)=\frac{4 \pi}{k_{0}^{2}}\left(J+\frac{1}{2}\right) \int_{0}^{k_{\max }} d k\left|N_{N \Delta}^{1 / 2}(k) T_{L_{\Delta} S_{\Delta}, L S}^{J}\left(k, k_{0}, E\right) \rho_{N N}^{1 / 2}(E)\right|^{2},
$$

where $k_{\max }$ can be calculated from Eq. (26) by setting $M=m_{\pi}+m_{N}$, and

$$
\begin{gathered}
\rho_{N N}(E)=\pi \frac{k_{0} E_{N}\left(k_{0}\right)}{2}, \\
N_{N \Delta}(k)=\pi k^{2} \frac{q E_{N}(q) E_{\pi}(q) E_{M}(k)}{M^{2}}\left|\frac{h_{\Delta \leftrightarrow \pi N}(q)}{E-E_{N}(k)-E_{\Delta}(k)-\Sigma(k, E)}\right|^{2} .
\end{gathered}
$$

To have a better understanding of Eq. (36), we use Eq. (25) to change the integration variable

$$
d k=-\frac{M E_{N}(k)}{k E} d M
$$

Eq. (36) can then be written as an integral over the invariant mass $M$ of the $\pi N$ subsystem

$$
d \sigma_{L_{\Delta} S_{\Delta}, L S}^{J}=\frac{4 \pi}{k_{0}^{2}}\left(J+\frac{1}{2}\right) \int_{m_{\pi}+m_{N}}^{E-m_{N}} d M\left|\Gamma_{N \Delta}(M)^{1 / 2} T_{L_{\Delta} S_{\Delta}, L S}^{J}\left(k, k_{0}, E\right) \rho_{N N}^{1 / 2}(E)\right|^{2} .
$$

where

$$
\begin{aligned}
\Gamma_{N \Delta}(M) & =\pi\left[\frac{k E_{N}(k) E_{M}(k)}{E}\right]\left[\frac{q E_{N}(q) E_{\pi}(q)}{M}\right] \\
& \times\left|\frac{h_{\Delta, \pi N}(q)}{E-E_{N}(k)-E_{\Delta}(k)-\Sigma(k, E)}\right|^{2} .
\end{aligned}
$$


Note that in evaluating the above integral, we need to use Eqs. (26) and (27) to express $k$ and $q$ in terms of the invariant mass $M$ and the total energy $E$. By Eqs. (26) and (34), the self-energy $\Sigma(k, E)$ is also a function of the total energy $E$ and the invariant mass $M$.

Equation (40) is an exact expression. The integrand contains the dependence on the invariant mass $M$ of the $\Delta^{*}, d \sigma_{L_{\Delta} S_{\Delta}, L S}^{J} / d M$, which is needed for a detailed transport-equation calculation. For practical applications and making contact with the current calculations of relativistic heavy-ion collisions, it is interesting to develop an averaging procedure such that the cross section for a given total energy $\mathrm{E}$ can be written in terms of only one averaged $\Delta^{*}$. This is our next task.

We use Eqs. (26) and (41) to define an average relative momentum $\bar{k}$ of the $N \Delta^{*}$ twobody system

$$
\bar{k}=\frac{\int_{m_{N}+m_{\pi}}^{E-m_{N}} \Gamma_{N \Delta}(M) k d M}{\int_{m_{N}+m_{\pi}}^{E-m_{N}} \Gamma_{N \Delta}(M) d M},
$$

The corresponding average mass of $\Delta^{*}$ is then calculated from Eq. (25)

$$
\bar{M}^{2}=E^{2}-2 E E_{N}(\bar{k})+m_{N}^{2} .
$$

Assuming that the transition t-matrix in the integrand of Eq. (40) can be calculated at the average momentum $\bar{k}$, we then obtain the following "factorized" form

$$
\sigma_{N \Delta^{*} \leftarrow N N}^{\text {tot }}=\sum_{J, L_{\Delta} S_{\Delta}, L S} d \bar{\sigma}_{L_{\Delta} S_{\Delta}, L S}^{J}
$$

with

$$
d \bar{\sigma}_{L_{\Delta} S_{\Delta}, L S}^{J}=\frac{4 \pi}{k_{0}^{2}}\left(J+\frac{1}{2}\right)\left|\rho_{N \Delta}^{1 / 2}(E) T_{L_{\Delta} S_{\Delta}, L S}^{J}\left(\bar{k}, k_{0}, E\right) \rho_{N N}^{1 / 2}(E)\right|^{2} .
$$

Here we have introduced a phase-space factor for the $N \Delta^{*}$ two-body state

$$
\rho_{N \Delta}(E)=\int_{m_{N}+m_{\pi}}^{E-m_{N}} d M \Gamma_{N \Delta}(M) .
$$

Equation (45) has the form similar to that introduced in the literature [6.5. Here we have explicitly shown how it can be derived from the Hamiltonian formulation [11 13] of the $\Delta$ 
excitation by using the "factorization approximation". $\Xi \Xi$ The accuracy of this approximation will be examined in section $\mathrm{V}$. We emphasize here that that $\Gamma_{N \Delta}(M)$, defined in Eq. (41), is the consequence of a unitary formulation of the $\pi N N$ processes listed in Eqs. (1)-(3). Equation (46) is significantly different from the parameterizations of the $N \Delta$ phase-space introduced in Refs. [4,5].

We note that Eq. (45) has the usual form for the binary collisions involving only stable particles. For example, the exact expression for $N N \rightarrow N N$ process can be obtained by replacing $\rho_{N \Delta}(E)$ in Eq. (45) by $\rho_{N N}(E)$.

By using the $N \Delta^{*}$ representation of the $\pi N N$ state, it is straightforward to carry out similar derivations to express the $\pi N N \rightarrow N N$ and $\pi N N \rightarrow \pi N N$ cross sections in terms of $N \Delta^{*} \rightarrow N N$ and $N \Delta^{*} \rightarrow N \Delta^{*}$ cross sections. If the averaging procedure defined in Eqs. (42) and (46) is also applied, we obtain the following expressions

$$
\sigma_{N N \leftarrow N \Delta^{*}}^{t o t}=\sum_{J, L S, L_{\Delta} S_{\Delta}} d \bar{\sigma}_{L S, L_{\Delta} S_{\Delta}}^{J}
$$

with

$$
d \bar{\sigma}_{L S, L_{\Delta} S_{\Delta}}^{J}=\frac{4 \pi}{\bar{k}^{2}}\left|\rho_{N N}^{1 / 2}(E) T_{L S, L_{\Delta} S_{\Delta}}^{J}\left(k_{0}, \bar{k}, E\right) \rho_{N \Delta}^{1 / 2}(E)\right|^{2},
$$

for the $N \Delta^{*} \rightarrow N N$ reaction, and

$$
\sigma_{N \Delta^{*} \leftarrow N \Delta^{*}}^{t o t}=\sum_{J, L_{\Delta}^{\prime} S_{\Delta}^{\prime}, L_{\Delta} S_{\Delta}} d \bar{\sigma}_{L_{\Delta}^{\prime} S_{\Delta}^{\prime}, L_{\Delta} S_{\Delta}}^{J}
$$

with

$$
d \bar{\sigma}_{L_{\Delta}^{\prime} S_{\Delta}^{\prime}, L_{\Delta} S_{\Delta}}^{J}=\frac{4 \pi}{\bar{k}^{2}}\left(J+\frac{1}{2}\right)\left|\rho_{N \Delta}^{1 / 2}(E) T_{L_{\Delta}^{\prime} S_{\Delta}^{\prime}, L_{\Delta} S_{\Delta}}^{J}(\bar{k}, \bar{k}, E) \rho_{N \Delta}^{1 / 2}(E)\right|^{2},
$$

for the $N \Delta^{*} \rightarrow N \Delta^{*}$ reaction.

We can further extend the above formula to calculate the differential cross sections involving a $\Delta^{*}$ resonance in either the entrance channel or the exit channel. Specifically, we can define a general scattering amplitude 


$$
\begin{aligned}
\tilde{T}_{\alpha \beta}\left(\vec{k}_{\alpha}, \vec{k}_{\beta}, E\right) & =\sum_{J M_{J}, L_{\alpha} S_{\alpha}, L_{\eta} S_{\beta}} y_{L_{\alpha} S_{\alpha}}^{J M_{J}}\left(\hat{k}_{\alpha}\right) y_{L_{\beta} S_{\beta}}^{J M_{J}^{+}}\left(\hat{k}_{\beta}\right) \\
& \times \rho_{\alpha}^{1 / 2}(E) T_{L_{\alpha} S_{\alpha}, L_{\beta} S_{\beta}}^{J}\left(\bar{k}_{\alpha}, \bar{k}_{\beta}, E\right) \rho_{\beta}^{1 / 2}(E),
\end{aligned}
$$

where $\alpha, \beta$ can be $N N$ or $N \Delta^{*}$ states, and $\left|\vec{k}_{\alpha}\right|=\bar{k}, k_{0}$ for $\alpha=N \Delta^{*}, N N$. The differential cross sections are then of the following form

$$
\frac{d \sigma_{\alpha \beta}(E)}{d \Omega}=\frac{16 \pi^{2}}{\bar{k}_{\beta}^{2}} \frac{1}{\left(2 s_{1_{\beta}}+1\right)\left(2 s_{2_{\beta}}+1\right)} \sum_{\text {spins }}\left|<m_{1_{\alpha}} m_{2_{\alpha}}\right| \tilde{T}_{\alpha \beta}\left(\vec{k}_{\alpha}, \vec{k}_{\beta}, E\right)\left|m_{1_{\beta}} m_{2_{\beta}}>\right|^{2}
$$

It is easy to show that the total cross sections Eqs. (44), (47), and (49) can be obtained by integrating Eq. (52) over the scattering angle $\Omega$.

\section{MEDIUM EFFECTS ON THE $\Delta$ PROPAGATION}

We follow the approach developed in Ref. [14] to calculate the medium effects on the $\Delta$ propagation. The resulting $\Delta$ potential is then used to calculate the medium effects on the scattering cross sections defined in the previous section.

In the one-hole-line approximation, the propagator of a $\Delta$ with momentum $\vec{p}_{\Delta}$ and energy $\epsilon_{\Delta}$ in nuclear matter can be written as

$$
G_{\Delta}\left(\vec{p}_{\Delta}, \epsilon_{\Delta}\right)=\frac{1}{\epsilon_{\Delta}-E_{\Delta}\left(\vec{p}_{\Delta}\right)-\Sigma^{(1)}\left(\vec{p}_{\Delta}, \epsilon_{\Delta}\right)-\Sigma^{(2)}\left(\vec{p}_{\Delta}, \epsilon_{\Delta}\right)},
$$

with

$$
\Sigma^{(1)}\left(\vec{p}_{\Delta}, \epsilon_{\Delta}\right)=\int \frac{Q_{\pi N}\left(k, p_{\Delta}\right)\left|h_{\pi N \leftrightarrow \Delta}(k)\right|^{2} k^{2} d k}{\epsilon_{\Delta}-\sqrt{\left(E_{N}(k)+E_{\pi}(k)\right)^{2}+p_{\Delta}^{2}}+i \epsilon}
$$

where $Q_{\pi N}\left(k, p_{\Delta}\right)$ is an angle-average Pauli operator [14] for the $\pi N$ state. The medium effect on the $\Delta$ propagation is calculated from the $N \Delta$ G-matrix

$$
\Sigma^{(2)}\left(\epsilon_{\Delta}, \vec{p}_{\Delta}\right)=\int_{p \leq p_{F}} d \vec{p}<\vec{p}_{\Delta} \vec{p}\left|G_{N \Delta, N \Delta}\left(W=\left(\epsilon_{\Delta}+\epsilon_{N}(\vec{p})\right)\right)\right| \vec{p}_{\Delta} \vec{p}>
$$

where $p_{F}$ is the Fermi momentum of nuclear matter.

It is convenient to calculate the $\mathrm{G}$ matrix in partial-wave representation. For symmetric nuclear matter( the total isospin and the total angular momentum are both equal to zero), we find that 


$$
\begin{aligned}
\Sigma^{(2)}\left(\vec{p}_{\Delta}, \epsilon_{\Delta}\right) & =\frac{1}{\left(2 s_{\Delta}+1\right)\left(2 \tau_{\Delta}+1\right)} \sum_{L_{\Delta} S_{\Delta} J T} \frac{(2 J+1)(2 T+1)}{4 \pi} \\
& \times \int_{p \leq p_{F}} d \vec{p} G_{L_{\Delta} S_{\Delta}, L_{\Delta} S_{\Delta}}^{J T}\left(q, q, \omega\left(\epsilon_{\Delta}, \vec{p}_{\Delta}, \vec{p}\right), P\right),
\end{aligned}
$$

where $q$ is the relative momentum, $\omega$ is the collision energy in the $N \Delta$ center of mass frame, and $P$ is the total momentum. In the nonrelativistic baryon approximation, we have

$$
\begin{gathered}
P=\left|\vec{p}_{\Delta}+\vec{p}\right|, \\
q=\left|\frac{m_{\Delta}^{0} \vec{p}-m_{N} \vec{p}_{\Delta}}{m_{\Delta}^{0}+m_{N}}\right|, \\
\omega\left(\epsilon_{\Delta}, \vec{p}_{\Delta}, \vec{p}\right)=\epsilon_{\Delta}+\epsilon_{N}(\vec{p})-m_{\Delta}^{0}-m_{N}-\frac{\left(\vec{p}_{\Delta}+\vec{p}\right)^{2}}{2\left(m_{\Delta}^{0}+m_{N}\right)},
\end{gathered}
$$

where $m_{\Delta}^{0}=1280 \mathrm{MeV}$ is the bare mass of the $\Delta$ determined from fitting the $\pi N$ phase shifts in $P_{33}$ channel.

The equations for the G-matrix are identical to Eqs. (6)-(9) except that the propagators are modified. In the partial-wave representation, each equation has the following structure

$$
\begin{aligned}
& G_{\alpha, \delta}^{J T}\left(q^{\prime}, q, \omega, P\right)=V_{\alpha, \delta}^{J T}\left(q^{\prime}, q, \omega\right) \\
& \quad+\sum_{\gamma} \int q^{\prime \prime 2} d q^{\prime \prime} \frac{V_{\alpha, \gamma}^{J T}\left(q^{\prime}, q^{\prime \prime}, \omega\right) Q_{\gamma}\left(q^{\prime \prime}, P, p_{F}\right)}{\omega-W_{\gamma}\left(q^{\prime \prime}, P\right)+i \epsilon} G_{\gamma, \delta}^{J T}\left(q^{\prime \prime}, q, \omega, P\right)
\end{aligned}
$$

where the greek subindices denote collectively the particle channels, $N N$ or $N \Delta$, and the orbital and spin quantum numbers. In the propagator of Eq. (57), $Q_{\gamma}\left(q^{\prime \prime}, P, p_{F}\right)$ is the angle-average Pauli operator [14] and

$$
W_{\gamma}(q, P)=\epsilon_{N}(\bar{p})+\epsilon_{N}(\bar{p})
$$

for $\gamma=N N$ channel, and

$$
W_{\gamma}(q)=\epsilon_{N}\left(\bar{p}_{N}\right)+\epsilon_{\Delta}\left(\bar{p}_{\Delta}\right)
$$

for $\gamma=N \Delta$ channel. The single particle energies in the above equations are calculated by using the angle-average momenta

$$
\bar{p}=\sqrt{q^{2}+\frac{1}{4} P^{2}},
$$


and

$$
\begin{aligned}
& \bar{p}_{N}=\sqrt{\left(\frac{m_{N} P}{m_{\Delta}^{0}+m_{N}}\right)^{2}+q^{2}}, \\
& \bar{p}_{\Delta}=\sqrt{\left(\frac{m_{\Delta} P}{m_{\Delta}^{0}+m_{N}}\right)^{2}+q^{2}} .
\end{aligned}
$$

The nucleon single particle energy $\epsilon_{N}(p)$ is taken from a variational many-body calculation by Wiringa [21]. The single particle energy for the $\Delta$ is determined by the following self-consistency condition

$$
\operatorname{Re}\left(\epsilon_{\Delta}\left(\vec{p}_{\Delta}\right)\right)=m_{\Delta}^{0}+\frac{p_{\Delta}^{2}}{2 m_{\Delta}^{0}}+\operatorname{Re}\left(\Sigma^{(1)}\left(\vec{p}_{\Delta}, \epsilon_{\Delta}\left(\vec{p}_{\Delta}\right)\right)+\operatorname{Re}\left(U_{\Delta}\left(\vec{p}_{\Delta}\right)\right)\right),
$$

where we have defined the $\Delta$ potential

$$
U_{\Delta}\left(\vec{p}_{\Delta}\right)=\Sigma^{(2)}\left(\vec{p}_{\Delta}, \epsilon_{\Delta}\left(\vec{p}_{\Delta}\right)\right)
$$

The medium effects on the cross sections of binary collisions defined in section III can be included by replacing the scattering t-matrix in Eqs. (45), (48), (50), and (51) by the G-matrix. The collision energy is calculated from real parts of the single particle energies $\epsilon_{N}(p)$ of Ref. [21] and $\epsilon_{\Delta}\left(p_{\Delta}\right)$ defined by the self consistent condition Eq. (60).

\section{RESULTS AND DISCUSSIONS}

We have applied the formula developed in section III to investigate the cross sections of binary collisions involving a $\Delta^{*}$ excitation in either the entrance channel or the exit channel. In Fig. 3, we show that the $N N \rightarrow N \Delta^{*}$ cross sections (dashed curve) calculated by using the factorized form Eqs. (44)-(45) can reproduce reasonably well the exact calculation of $N N \rightarrow \pi N N$ using Eqs. (35) and (40). This suggests that the factorized forms defined by Eqs. (44)-(52) are sufficient for investigating the main features of relativistic heavy-ion collisions. The calculated total cross sections for all processes involving $\Delta^{*}$ are compared in Fig. 4. They have quite different energy dependencies. The difference between $N N \rightarrow N \Delta^{*}$

and $N \Delta^{*} \rightarrow N N$ is due to the flux factors $1 / k_{0}^{2}$ of Eq. (45) and $1 / \bar{k}^{2}$ of Eq. (48). This can be understood from Fig. 5 in which the calculated $\bar{k}$ for the $N \Delta^{*}$ channel is compared with the 
momentum $k_{0}$ of the $N N$ channel. The calculated mass of the $\Delta^{*}$ is also displayed there. We see that as the collision energy increases the produced $\Delta^{*}$ becomes heavier and moves faster.

The predicted differential cross sections at several energies are compared in Fig. 6. We see that the angular distributions for $N N \rightarrow N \Delta^{*}$ is quite different from that of $N \Delta^{*} \rightarrow N N$. This is due to the fact that the important partial waves in $N N$ and $N \Delta$ are quite different. For example, in the $\mathrm{J}=2$ channel the outgoing particles are in ${ }^{1} D_{2}$ for the $N \Delta^{*} \rightarrow N N$, but are in ${ }^{5} S_{2}$ for the $N N \rightarrow N \Delta$. Clearly such a dynamical difference in differential cross sections can not be easily obtained by using detailed balance [3] to relate $N \Delta^{*} \rightarrow N N$ to the data of the $N N \rightarrow N N \pi$ reaction. We further notice that the predicted $N \Delta^{*} \rightarrow N \Delta^{*}$ differential cross sections are forward peaked. This is also quite different from the usual assumption that $N \Delta^{*} \rightarrow N \Delta^{*}$ is identical to $N N \rightarrow N N$ apart from some isospin factors. The results shown in Figs. 4 and 6 are the consequences of the considered meson-exchange mechanisms.

To get some ideas about the medium effects on collision cross sections, we assume that the mean field effect on the $\Delta$ propagation is the potential $U_{\Delta}$ defined in Eqs. (56) and (61). The calculated $U_{\Delta}$ at several densities are displayed in Fig. 7. Both the real parts and imaginary parts depend strongly on the density and the momentum. Qualitatively, our results indicate that the $\Delta$ moves almost freely in nuclear medium at high momentum, but is slowed down considerably and is easily annihilated at low momentum. It will be interesting to explore the consequence of the predicted $U_{\Delta}$ in determining the pion yields in relativistic heavy-ion collisions.

The predicted $U_{\Delta}(p)$ is used to evaluate the $\Delta$ single particle energy Eq. (60) and the $\mathrm{G}$ matrix elements which are the inputs to the calculations of the cross sections in medium, as explained in section IV. We have found that the medium effects are most dramatic in the region where the collision energies are close to the $\pi N N$ threshold in free space. This can be illustrated in a calculation where a $\Delta^{*}$ with a mass of $1236 \mathrm{MeV}$ propagates under the influence of the mean field $U_{\Delta}$ and collides with a nucleon at rest in nuclear matter. 
The predicted dependence of the $N \Delta^{*} \rightarrow N N$ and $N \Delta^{*} \rightarrow N \Delta^{*}$ total cross sections on the density and the momentum is displayed in Fig. 8. There are two medium effects. The first one is the mean field effect on the propagators of the G-matrix in Eq. (57). This effect is found to be not very large in the momentum region considered. The main medium effect is due to the change of the collision energies by the $\Delta$ potential $U_{\Delta}$ in the entrance channels. Since the potential energy(real part) becomes more attractive as density increases (Fig. 7), the collision energy for a given momentum of the incident $\Delta$ is shifted to the lower energy region closer to the pion production threshold where the cross sections vary rapidly as shown in Fig. 4. We therefore see in Fig. 8 that the $N \Delta^{*}$ collision cross sections in the low momentum region depend strongly on the density, and are greatly suppressed at high densities. A similar situation is also found for $N N \rightarrow N \Delta^{*}$, as illustrated in the lower half of Fig. 9. The corresponding medium effects on the $N N \rightarrow N N$ are less dramatic since the $N N$ elastic cross sections do not vary so rapidly in the energy region near pion production threshold.

\section{SUMMARY}

The binary collisions involving a $\Delta$ resonance in either the entrance channel or the exit channel have been investigated within a Hamiltonian formulation of $\pi N N$ interactions [11 13. An averaging procedure has been developed to define the experimentally measured $N N \rightarrow \pi N N$ cross section in terms of an effective $N N \rightarrow N \Delta^{*}$ cross section. In contrast to previous works [4,5], the main feature of the present approach is that the mass and the momentum of the produced $\Delta^{*}$ at each collision energy are calculated dynamically from the bare $\Delta \leftrightarrow \pi N$ vertex interaction of the model Hamiltonian and are constrained by the unitarity condition. The procedure is then extended to define the effective cross sections for the experimentally inaccessible $N \Delta^{*} \rightarrow N N$ and $N \Delta^{*} \rightarrow N \Delta^{*}$ reactions. The calculations have been performed to predict the energy dependencies and angular distributions of these cross sections. 
The $\Delta$ mean field in nuclear matter has been calculated by using the Bruckner-HartreeFock approximation developed in [14]. It is found that the $\Delta$ moves almost freely at high momenta, but is slowed down considerably and get easily absorbed at low momenta. By including the medium effect on the $\Delta$ propagation, the dependence of the effective cross sections of the $N \Delta^{*}$ collisions on the density has been investigated. It is demonstrated that the density dependence is most dramatic in the energy region close to the pion production threshold.

Our results can be used in the relativistic heavy-ion calculations using transport equations [3] 9]. To make further progress in investigating $\Delta$-rich matter, it is necessary to investigate the $\Delta^{*} \Delta^{*}$ collisions and the effects due to the higher mass $N^{*}$ resonances. This requires the extension of the $\pi N N$ model Hamiltonian defined by Eqs. (4)-(5) to include the two $-\pi$ channels and the development of a scattering theory with $\pi \pi N N$ unitary condition. Such a Hamiltonian model can be accurately constructed only when the dynamics of our present understanding of the $N^{*}$ excitations in $\pi N$ and $\gamma N$ reactions is improved quantitatively. The proposed pion facility at GSI [2] and the $N^{*}$ program at CEBAF are essential for making progress in this direction.

This work is supported by the U.S. Department of Energy, Nuclear Physics Division, under contract W-31-109-ENG-38. 


\section{REFERENCES}

[1] U. Mosel and V. Metag, Nucl. Phys. News 3 (1993) 25.

[2] R. Averbeck, A. Döppenschmidt, V. Metag, and R. S. Simon, GSI-Nachrichten 10 - 95 (1995) 15.

[3] G. F. Bertsch and S. Das Gupta, Physics Report 160 (1988) 189.

[4] P. Danielewicz and G. F. Bertsch, Nucl. Phys. A533 (1991) 712.

[5] Gy. Wolf, B.Batko, W. Cassing, U. Mosel, K. Niita, and M. Schäfer, Nucl. Phys. 517 (1990) 615 .

[6] Gy. Wolf, W. Cassing, and U. Mosel, Nucl. Phys. A552 (1993) 549.

[7] B.A. Li and W. Bauer, Phys. Rev. C44 (1991) 450.

[8] L. Xiong. Z.G. Wu, C.M. Ko, and J.Q. Wu, Nucl. Phys. A512 (1990) 772.

[9] C. Muntz et al., Z. Phys. A352 (1995) 175.

[10] J. R. Jeukenne, A. Lejeune, and C. Mahaux, Phys. Repo. 25 (1976) 83.

[11] T.-S. H. Lee, Phys. Rev. Lett. 20 (1983) 1571; Phys. Rev. C29 (1984) 195.

[12] T.-S. H. Lee and A. Matsuyama, Phys. Rev. C32 (1985) 516; Phys. Rev. C36 (1987) 1459.

[13] A. Matsuyama and T.-S. H. Lee, Phys. Rev. C34 (1986) 1900; Nucl. Phys. A526 (1991) 547.

[14] T.-S. H. Lee and K. Ohta, Phys. Rev. C25 (1982) 3043.

[15] V. Carlson et al., ANL-HEP-PR-95-64 preprint, Argonne National Laboratory, (1995).

[16] H. Garcilazo and T. Mizutani, $\pi N N$ System, World Scientific (1990).

[17] M. Betz and T.-S. H. Lee, Phys. Rev. C23 (1981) 375. 
[18] G.-H. Niepleaus, M. Gari, and B. Sommer, Phys. Rev. C20 (1979) 1096.

[19] M. Lacombe, B. Loiseau, J. M. Richard, R. Vinh Mau, J. Cott, P. Pine, and K. Tourreil, Phys. Rev. C21 (1980) 861.

[20] H. Popping, P. U. Sauer, and X.Z. Zhang, Nucl. Phys. A474 (1987) 557.

[21] R. B. Wiringa, Phys. Rev. C38 (1988) 1010.

[22] B. J. VerWest and R. A. Arndt, Phys. Rev. C25 (1982) 1979. 


\section{FIGURES}

FIG. 1. The polarization observables $C_{L L}$ and $C_{L S}$ of np scattering predicted by the $\pi N N$ model [11 13] are compared with the recent data [15].

FIG. 2. Graphical representation of the $N N \rightarrow N \Delta \rightarrow \pi N N$ reaction.

FIG. 3. The calculated $N N \rightarrow \pi N N$ (solid curve) and $N N \rightarrow N \Delta^{*}$ (dotted curve) total cross sections are compared. The data are from the compilation of Ref. [22].

FIG. 4. The predicted total cross sections for the binary collisions involving a $\Delta^{*}$ in either the entrance channel or the exit channel are compared.

FIG. 5. The mass $\bar{M}^{*}$ and momentum $\bar{k}$ of the $\Delta^{*}$ in the $N N \rightarrow N \Delta^{*}$ reaction. $E_{L}$ is the incident nucleon energy in the laboratory frame.

FIG. 6. The predicted angular distributions evaluated at energies (in terms of the corresponding nucleon laboratory energies) $E_{L}=400 \mathrm{MeV}$ (dotted curves), $700 \mathrm{MeV}$ (solid curves), and $900 \mathrm{MeV}$ (dashed curves).

FIG. 7. The calculated $\Delta$ potentia $U_{\Delta}\left(p_{\Delta}\right)$ at several densities. The density $\rho$ is in units of $\rho_{0}=0.16$ nucleons- $\mathrm{fm}^{-3}$.

FIG. 8. The momentum-dependence of the calculated total cross sections of $N \Delta^{*} \rightarrow N N$ and $N \Delta^{*} \rightarrow N \Delta^{*}$ collisions in nuclear matter. The density $\rho$ is in units of $\rho_{0}=0.16$ nucleons-fm ${ }^{-3}$.

FIG. 9. The momentum-dependence of the calculated $N N \rightarrow N N$ and $N N \rightarrow N \Delta^{*}$ collisions in nuclear matter. The density $\rho$ is in unit of $\rho_{0}=0.16$ nucleons-fm $^{-3}$. 